\title{
Regional Situation Awareness in Complex Terrain Using Spaceborne Video
}

\section{SAR}

\author{
Liang Bian ${ }^{a}$, Jing-Wen Lii ${ }^{b}$, Wei Yang ${ }^{c, *}$ \\ School of Electronics and Information Engineering, BeiHang University, XueYuan Road NO.37, BeiJing, \\ China \\ a13466713113@163.com, ${ }^{b}$ lijingwen@buaa.edu.cn, c yangweigigi@sina.com
}

Keywords: Situation Awareness, SAR, Video, Complex Terrain

\begin{abstract}
Synthetic Aperture Radar (SAR) is one of the active microwave imaging sensors, which can be work all-day and all weather. However, the current SAR system cannot provide the continuous surveillance, limiting the application of SAR images, especially in the case of complex terrain. In this paper, the new imaging modes are proposed based on video SAR, which is suitable for regional situation awareness in complex terrain. Then, the performance of Video SAR system is analysed combined with the imaging mode. Moreover, a parallel imaging method based on DCS algorithm is presented for video SAR data fast focusing. Finally, experimental results are provided to demonstrate the effectiveness of the proposed method.
\end{abstract}

\section{Introduction}

Synthetic aperture radar (SAR) images have been widely used in many areas, such as target recognition, disaster rescue and environmental monitoring [1]. And, several state-of-the-art imaging modes have been proposed benefited from the azimuth antenna beam steering technique, such as the TOPS, sliding spotlight and staring spotlight modes [2]. However, the modes mentioned above can just provide static images, without the capability for dynamically change information.

In order to improve the performance for regional situation awareness in complex terrain, the video SAR mode is developed, drawing on the experience of optical video [3]. The concept of video SAR is firstly proposed by Sandia National Laboratories in 2005, which illustrates the working mechanism. Then, DARPA (The Defense Advanced Research Projects Agency of America) has raised a development plan for video SAR in 2012, which discusses the spatial resolution and coverage performance. As for the imaging processing of video SAR, back-projection algorithm is proposed combined with a sub-aperture circular shift register in [4], which forms a new frame image using the processing results of the previous frame image, improving the processing efficiency significantly. Moreover, in order to further increasing the processing speed of video SAR product, several parallel algorithm methods have been developed, by introducing the GPU (Graphics Processing Units) for acceleration calculation.

At present, some airborne Video SAR systems have been already developed, as well as the experiment results. However, the observation coverage area of airborne Video SAR is relatively small, and it is easily to be affected by weather condition and environment. Therefore, it is not suitable for regional situation awareness, especially in the case of complex terrain. Compared with the airborne Video SAR, spaceborne Video SAR has obvious advantages, such as the quicker response and wider swath. But, the research of spaceborne video SAR is still in the early stage, which needs more in-depth study.

In this paper, the spaceborne video SAR is studied for regional situation awareness in complex terrain. First, the new imaging modes are proposed, as well as the discussion of performance. Then, a parallel imaging method is presented for video SAR data fast focusing. Finally, the experimental results are discussed and the conclusions are given. 


\section{Video SAR Imaging Mode}

Video SAR can provide video products, which is different from the traditional SAR system. Consequently, the imaging modes of Video SAR are also distinct. Aim at the regional situation awareness, three imaging modes of spaceborne SAR are proposed, namely the tracking video mode, spotlighting video mode, and the surrounding video mode.

\subsection{Tracking Video SAR Mode}

Tracking video SAR mode is proposed for moving target monitoring. As shown in Figure.1, by adopting the beam steering technique, the satellite scans the strip-map region multi-times. Therefore, the moving target can be observed continuously. Furthermore, the velocity of moving target also can be estimated based on tracking video product.

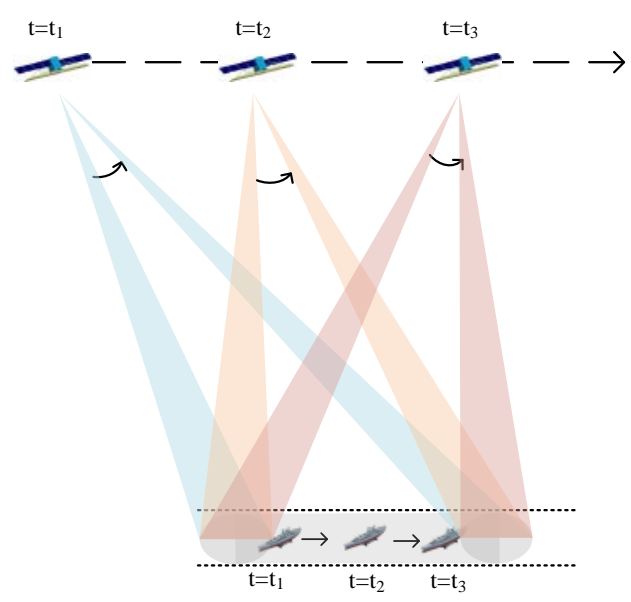

Figure 1 Tracking video SAR mode.

\subsection{Spotlighting Video SAR Mode}

Spotlighting video SAR mode is designed for target area fine observation. In this mode, the azimuth antenna beam is steered from forward to backward, which means that the target area can be observed with different angles, as shown in Figure.2. Therefore, the video product can be provided for illustrating the target from different angles, which is more suitable for users to monitor the target area.

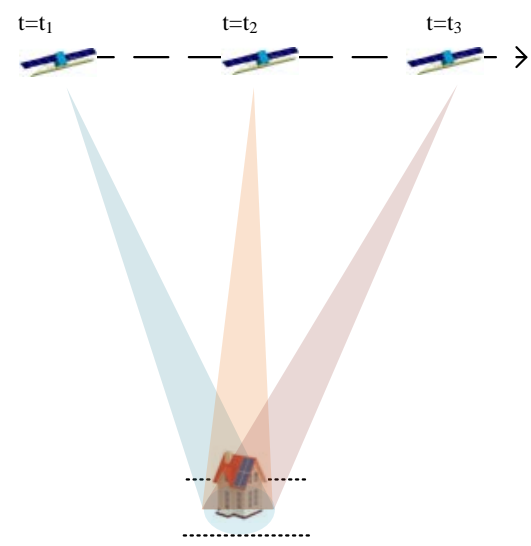

Figure 2 Spotlighting video SAR mode.

\subsection{Surrounding Video SAR Mode}

Different from the tracking video SAR mode and spotlighting video SAR mode, which can be realized by satellite single-pass, the surrounding video SAR mode need to be realized by multi-pass combined with SAR left-side observation and right-side observation. Using the surrounding video SAR mode, the target area can be observed from different aspects, which is illustrated in Figure. 3. 
Consequently, the occlusion target has greater chance to be detected, especially in the case of the target behind the building or buildings hidden behind the mountain. Moreover, by Post-processing, the video product that can describe the area in detail is obtained. So, this mode is very suitable for regional situation awareness in complex terrain.

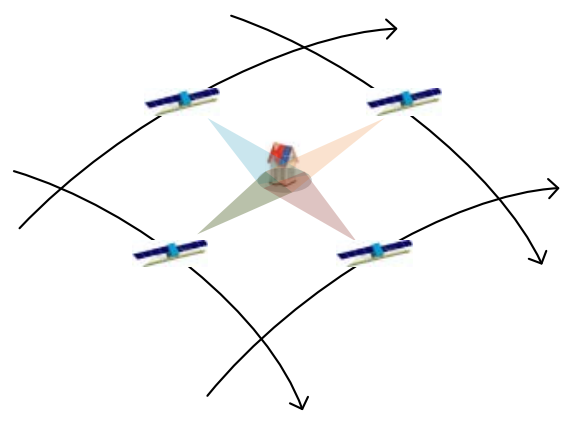

Figure 3 Surrounding video SAR mode.

\section{System Performance Analysis}

In this section, several core performance parameters are analysed, including the data overlapping rate, resolution, observation time, and frame rate, which is important for system design.

\subsection{Data Overlapping Rate}

In order to obtained the video product, more images should be obtained to form video. Therefore, as for the video SAR, the echo data should be firstly divided into sequential segments with two methods, as shown in Figure. 4.

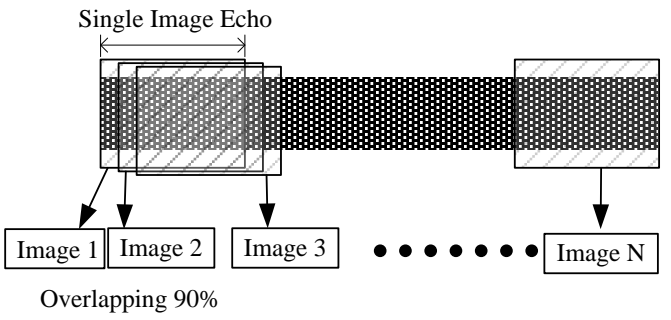

a. Data overlapping (90\%)

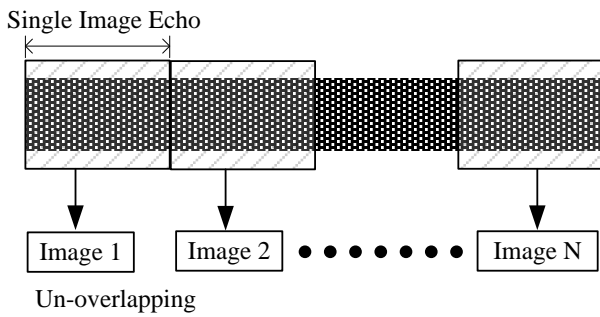

b. Data un-overlapping

Figure 4 Data division methods of spaceborne Video SAR.

The first division method is implemented with echo data overlapping. Since every data segment can be processed to form an image, more images can be obtained using the overlapping method. Compared with the first division method, the second method is performed without overlapping, which means smaller images can be obtained. However, with respect to the overlapping method, the redundant information between adjacent images is larger. To describe the degree of date overlapping, the data overlapping rate is defined as follow:

$$
\gamma=\frac{N_{a}-\Delta N_{a}}{N_{a}}
$$

where $N_{a}$ is the azimuth point number of data segment, and $\Delta N_{a}$ is azimuth new added point.

\subsection{Resolution}

Resolution is one of the most important indicators of SAR images, which affects the application efficiency directly. As for the video SAR imaging modes mentioned above, the range resolution and azimuth resolution can be calculated by 


$$
\begin{gathered}
\rho_{r}=\frac{c}{2 \cdot B_{r} \cdot \sin \eta} \\
\rho_{a}=\frac{\lambda}{2\left(\sin \theta_{i}-\sin \theta_{i-1}\right)}
\end{gathered}
$$

where $B_{r}$ is the bandwidth of radar transmit signal, $c$ is the speed of light, $\eta$ is the angle of incidence, $\lambda$ is the wavelength, $\theta_{i-1}$ and $\theta_{i}$ the starting angle and the ending angle correspond to the echo data segment $i$.

\subsection{Observation Time}

According to the geometry mode showed in Figure. 1 to Figure. 3, the duration of the video can be represented as

$$
T_{a}=\frac{\left(R_{0} \tan \alpha_{e}-R_{0} \tan \alpha_{s}\right)}{V_{s}}
$$

where $R_{o}$ is the closest approach distance, $\alpha_{s}$ is the starting angle, $\alpha_{e}$ is the ending angle, and $V_{s}$ is the velocity of the satellite.

\subsection{Frame Rate}

Frame rate is a special parameter for spaceborne Video SAR compared with traditional SAR. According to the two division methods mentioned above, frame rate is analysed respectively. For un-overlapping method, the synthetic aperture length that corresponds to the segment $i$ can be represented as

$$
L=R_{m}\left(\sin \theta_{i}-\sin \theta_{i-1}\right)
$$

where $R_{m}$ is the slant range. So, the duration time is given by

Thus, the frame rate is

$$
t_{\text {ceil }}=\frac{L}{V_{s}} \approx \frac{R_{m} \lambda}{2 V_{s} \rho_{a}}
$$

$$
f_{p s}=\frac{1}{t_{\text {ceil }}}=\frac{2 V_{s} \rho_{a} f_{0}}{R_{m} c}
$$

With respect to the overlapping case, the overlapping ratio between two neighboring segments is $\gamma$. Thus, the duration time for segment $i$ is

And, the frame rate is given by (9)

$$
t_{\text {ceil }}=\frac{L}{V_{s}} \approx \frac{R_{m} \lambda}{2 V_{s} \rho_{a}}(1-\gamma)
$$

$$
f_{p s}=\frac{2 V_{s} \rho_{a} f_{0}}{R_{m} c(1-\gamma)}
$$

\section{Video SAR Image formation}

This section is focused on the Image formation for spaceborne video SAR. A modified DCS (Deramp Chirp Scaling) algorithm is proposed, which is shown in Figure. 5, including the part of echo division, deramp operation, chirp scaling (CS), and Fusion. Moreover, in order to improve the processing efficiency, the data segments can be processed in parallel. 


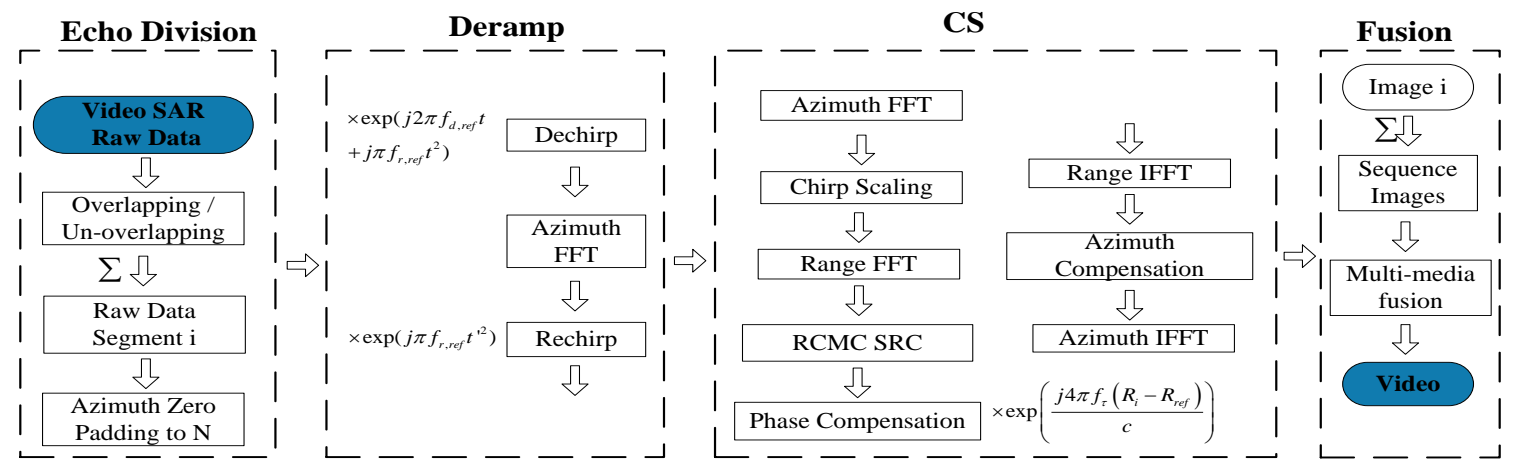

Figure 5 Flowchart of the modified DCS algorithm for spaceborne video SAR

According to the requirement of frame rate, the date overlapping rate is determined, and the echo division can be implemented. Then, the Deramp operation and CS algorithm are introduced for image focusing [5]. Finally, the video product is obtained using sequence images.

To verify the processing method, numerical simulations are implemented. As shown in Figure 6, the simulation scene is designed with point A moving along range direction at a speed of $5 \mathrm{~m} / \mathrm{s}$, point $C$ along azimuth direction with a speed of $10 \mathrm{~m} / \mathrm{s}$ and point $B$ is motionless. By echo data simulation and image processing, the image formation results correspond to different frames are shown in Figure 7.

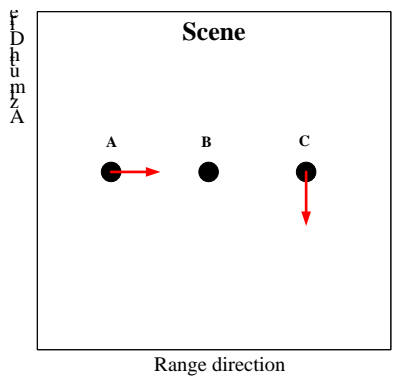

Figure 6 Point targets to be simulated

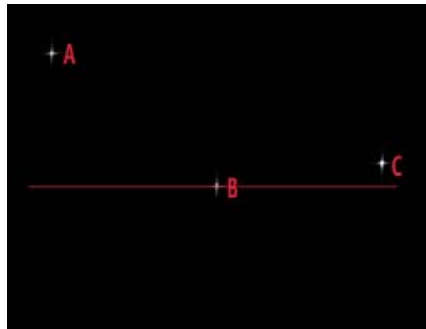

a. Frame 0

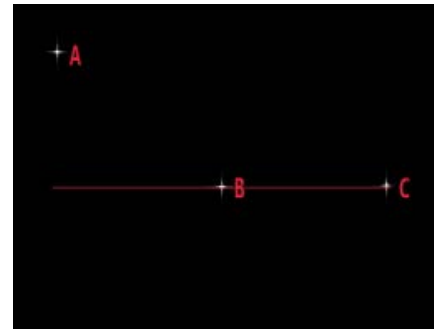

b. Frame 20

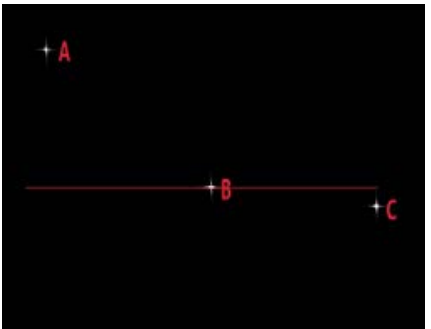

c. Frame 40

Figure 7 Simulation results

\section{Conclusion (Heading 3)}

In this paper, the new imaging modes are proposed based on video SAR for regional situation awareness, including the tracking video mode, spotlight video mode and the surrounding video mode. Based on these modes, the system performance is analysed, especially the discussion of frame rate. Moreover, the image formation method is addressed based on the DCS algorithm. Finally, the effectiveness of the proposed method is demonstrated by simulation results.

\section{Acknowledgements}

This work is supported by NSFC (Grant Nos. 61701012). 


\section{References}

[1] Janoth, J., GmbH, I., Gantert, S., Koppe, W., Kaptein, A. and Fischer, C. (2012) “TerraSARX2 - Mission overview,” IEEE Geosci. Remote Sens. Symp., Munich, Germany, Jul. 22-27.

[2] Chen, J., kuang, H., Yang, W., Liu, W. and Wang, P.B. (2016) “A novel imaging algorithm for focusing high-resolution spaceborne SAR data in squinted sliding-spotlight mode,” IEEE Geosci. Remote Sens. Lett., vol. 13, no. 10, 1577-1581.

[3] Wells, L., and Sorensen, K. (2005) "Developments in SAR and IFSAR systems and technologies at sandia national laboratories”, Aerospace Conferences, 1-4

[4] Miller, J., Bishop, E., and Doerry A. (2013) “An Application of Backprojection for Video SAR Image FormationExploiting a Subaperture Circular Shift Register”, 1-14.

[5] Yang, W., Chen, J., Zeng, H., Wang, P. and Li, C. (2013) “A novel three-step image formation scheme for unified focusing on spaceborne SAR data,” Progr. Electromagn. Res., 621-642. 